\title{
Article
}

\section{Battery life estimation of a battery under different stress condi- tions}

\author{
Natascia Andrenacci ${ }^{1, *}$, Francesco Vellucci ${ }^{1}$ and Vincenzo Sglavo ${ }^{1}$ \\ 1 C.R. ENEA Casaccia, via Anguillarese 301, 00123 Rome (Italy) \\ 2 Affiliation 2; e-mail@e-mail.com \\ * Correspondence: natascia.andrenacci@enea.it.
}

\begin{abstract}
The prediction of capacity degradation, and more generally of the behaviors related to battery aging, is useful in the design and use phases of a battery to help improve the efficiency and reliability of energy systems. In this paper, a stochastic model for the prediction of battery cell degradation is presented. The proposed model takes its cue from an approach based on Markov chains, although it is not comparable to a Markov process, as the transition probabilities vary as the number of cycles that the cell has performed varies. The proposed model can reproduce the abrupt decrease in the capacity that occurs near the end of life condition ( $80 \%$ of the nominal value of the capacity) for the cells analyzed. Furthermore, we illustrate the ability of this model to predict the capacity trend for a lithium-ion cell with nickel-manganese-cobalt (NMC) at the cathode and graphite at the anode subjected to a life cycle in which there are different aging factors, using the results obtained for cells subjected to single aging factors.
\end{abstract}

Keywords: cycle aging; Lithium battery; stochastic algorithm.

\section{Introduction}

Over the past two decades, batteries have become an important part of our lifestyle: from portable electronics to electric vehicles, to the recent integration of renewable energy into the power grid. In addition to the results obtained with the aim of increasing energy and power density, much effort has been made to understand the intrinsic degradation processes that occur during the service life, leading to a continuous deterioration of the health of the cell battery, such as loss of capacity or increase in internal resistance. Understanding the impact of the aging factors is also essential for developing reliable diagnostic and prognostic tools. However, predicting the battery aging trajectory is technically difficult because battery degradation is a complex non-linear process with coupled physical and chemical reactions and battery life is highly dependent on application (cycle life) or storage conditions (calendar life) [1]. One of the simplest solutions to obtain the battery capacity degradation trajectory is to conduct direct experiments in a specific load or storage condition. However, this solution generally requires a rather long experimental time of several months or even years. On the other hand, the accelerated tests must be appropriately designed to extrapolate the aging of cells under normal conditions, according to non-trivial protocols [2].

Battery degradation is affected by many factors, both at the individual cell level and the assembly level, and their effects are often correlated [3]. As the system is assembled, starting from single cells to modules and battery packs, the possible causes of stress for the batteries increase and their coupling intensifies [4]. It is therefore essential to understand the phenomenon of aging as early as possible in the complexity chain $[5,6]$. 
At the single-cell level, the degradation factors have thermodynamic and kinetic origins. Changes in the initial cell properties are influenced by the environment (e.g. temperature or pressure) and the duty cycle (e.g. voltage, current intensity, etc.) [7, 8]. Batteries deteriorate even when not in use (so-called "calendar life") and storage conditions, mainly temperature and state of charge (SOC), affect the rate of degradation and battery performance [9].

Cycle aging refers to aging due to the continuous charge/discharge cycle of the battery. Calendar aging inevitably occurs during the life of the battery, regardless of the operating mode, and all factors of calendar aging also affect cyclic aging, especially when the battery duty cycle is made up of work phases alternating with rest phases [10]. Cycle life, however, is influenced by additional factors, such as the intensity of the current and the depth of discharge of the cycles. These aging factors act in such a way that the effects are not linearly correlated, which greatly complicates the understanding and description of the aging process. In particular, the temperature represents a very relevant factor in the degradation of the batteries, both in the operating conditions defined by the battery manufacturer, $[11,12]$ and in the case of uncontrolled temperature conditions.[12] Since this aging factor has been extensively covered in the literature, in the present work, the temperature remains constant during the life tests, to emphasize the effects of the other stress factors.

The article is structured as follows: Chapter 2 illustrates the main battery degradation mechanisms and the most popular modeling approaches. Chapter 3 describes the setup of the experimental tests and introduces the model proposed for the degradation of battery capacity. Finally, the main conclusions are summarized in Chapter 4.

\section{Battery ageing and modeling}

There are different mechanisms of aging and to facilitate their understanding and interpretation, they are commonly grouped into three different modes of degradation:

- $\quad$ Loss of conductivity (CL);

- Loss of active material (LAM), due to the degradation processes in which the use of the active electrode material is reduced;

- $\quad$ Loss of the lithium supply (LLI), caused by side reactions that irreversibly consume a portion of the lithium available in the cell.

CL includes the degradation of the electronic parts of the battery, such as corrosion of the current collector or decomposition of the binder. LAM is related to the structural transformations of the active material and the decomposition of electrolytes, such as the oxidation of the electrolyte, the intercalation gradient in the active particles, and the disturbance of the crystal structure. The LLI is attributed to the change in the number of lithium ions available for the electrochemical cell intercalation and de-intercalation processes, electrolyte decomposition, lithium plating, and lithium-ion granule formation. Some degradation mechanisms are caused by the mechanical action of the displacement, intercalation, and de-intercalation of the ions: in electrode-active materials, the insertion/extraction of lithium ions is, in general, accompanied by local deformation and volume variation of the materials. Due to the inherent non-equilibrium working conditions of the cells, this deformation is inhomogeneous in the materials and causes high internal stress which ultimately leads to fracture, fragmentation, or pulverization. Due to a loss of contact with conductive agents or electrolytes, the active materials can become partially inactive, thus contributing to the loss of overall capacity and loss of power and promoting fracture or delamination at the interface [1, 7-8].

The degradation can be measured through the progressive loss of capacity (which results in a decrease in autonomy) and the increase in internal resistance, which leads to 
a decrease in the power delivered. The fact that the performance losses are progressive poses the problem of the definition of end-of-life (EOL, End of Life) for Li-ion batteries. In the present work, the EOL condition is set at the $20 \%$ reduction of the initial capacity following the ISO 12405-2 standard "Electrically propelled road vehicles - Test specification for lithium-ion traction battery packs and systems - Part 2: High-energy applications " [13].

Battery cell-level aging analysis is one of the key aspects for the successful and reliable design of large-format energy storage systems based on lithium-ion technology. Ideally, the aging model should be valid for any real condition. The model must be developed based on the analysis of the different causes that affect aging, i.e. stress or impact factors such as SOC, temperature, DOD, current rate (Crate), and Ampere-hour throughput (Ahthroughput) or the number of cycles, appropriately defined.

To obtain effective battery aging predictions that can be used also for online prediction applications, the starting point is thus the experimental degradation trajectories, to which various methods can be applied to extract the battery degradation tendency over time so that future predictions can be made with a reasonable degree of confidence. There are many algorithms for these predictions, such as those based on artificial intelligence or on filters, which are very useful in online forecasts [14].

An effective type of approach is represented by the class of models based on the physics and chemistry of the storage systems, the so-called electrochemical models. They make use of different partial differential equations systems to model the physicochemical phenomena that occur in the battery and directly explain the aging behavior of the same. In most cases, they refer to the works of Doyle, Fuller and Newman [15-17], in which charge and discharge phenomena are modelled [15] along with the relaxation phenomena of lithium insertion processes [17], making use of the theory of concentrated solutions. These models are usually very accurate, but they are also generally very computationally expensive [18].

Analytical approaches are also quite widespread. Equivalent circuit models are among the most popular, since they have a level of accuracy that can be satisfactory, a lower complexity than electrochemical models and much shorter simulation times.

The simplest equivalent circuit is an ideal voltage source and resistance. However, the accuracy of this model is very low. If a capacity-resistance (RC) system is added, the model can reproduce the battery polarization effects, becoming more reliable. These types of equivalent circuits are called "Thevenin models". Adding further RC networks to the equivalent circuit, the models become more adherent to reality, but the complexity of resolution increases [18].

Alternatively, empirical models such as the single exponential model, the double exponential model, the linear model, or the polynomial model are often adopted [19, 20]. Common to these empirical models is an explicit mathematical form and ease of implementation, however, these models tend to be sensitive to noise, especially when training data are limited.

Statistical approaches rely heavily on experimental data to predict battery behaviour. Stochastic models describe the battery in an abstract way, as in analytical models. An example of statistical approach is the Autoregressive Moving Average (ARMA), which uses time series to deduce the aging level [21]. Particle filtering is another possible statistical approach that can predict the trend of the battery degradation [22].

The Bayesan approach to failure theory can also be applied to the EOL estimation, and the Weibull law is among the most used formula [23]. The Markov chains can also be used to describe the aging phenomenon, in particular they can reproduce charge recovery and capacity recovery (an effect that implies a partial recovery of battery capacity and that can occur during a period of inactivity in the charge and discharge phase) and Peukert's law [24]. 
In the present work, we have taken into consideration a model inspired by the theory of Markov chains and, based on the collected data, we have verified its prediction accuracy for NMC-graphite lithium-ion cells.

\section{Experimental set-up and modeling}

In this chapter we illustrate the principal characteristics of the experimental set up and the tests conducted, and introduce the model used to interpretation of the data.

\subsection{Life tests for lithium-ion cells}

The purpose of the tests is to investigate the effect of some external factors on battery life. In particular, life tests were performed by varying the intensity of the discharge current and the depth of discharge. The tests are performed on EiG PLB C020 20Ah cells, which are lithium-ion-polymer batteries with a cathode NMC technology, graphite at the anode, and a pouch structure (see Figure 1).

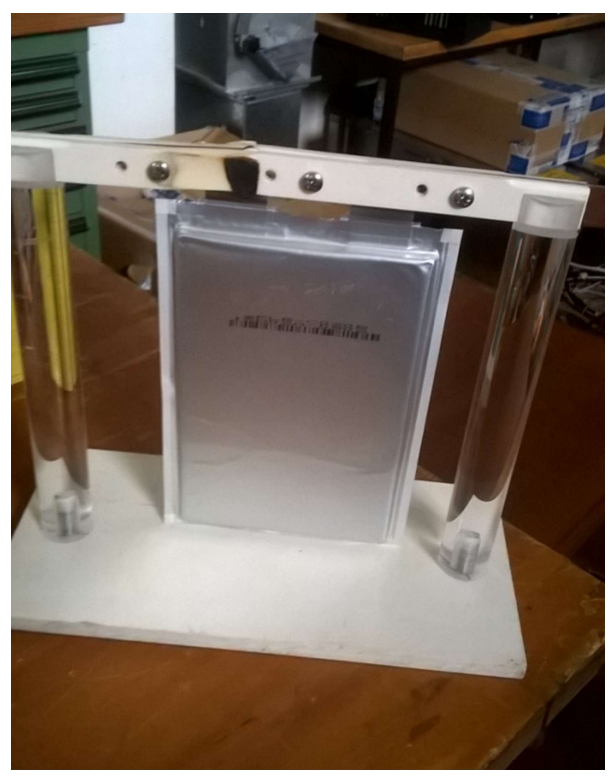

Figure 1: EiG PLB C020 cell.

The ENEA battery measurements campaign was launched in 2015, and the equipment used and the experimental protocols proposed are briefly described below.

The main electrical and operating characteristics of the cells are shown in Table 1 . The cycle life of these cells is declared as 1000 cycle at 250C, with a depth of discharge (DoD) of $100 \%$ and a current rate for charge and discharge of $1 \mathrm{C}$. The condition for the end of life corresponds to a $20 \%$ decrease of the capacity to the nominal value. All the parameters of our tests fall within the limits indicated by the manufacturer in the datasheet.

Table 1: Main electric and operating characteristic of EiG PLB C020 cells.

\begin{tabular}{|l|l|}
\hline Cell characteristic & Value \\
\hline Nominal voltage & $3.65 \mathrm{~V}$ \\
\hline Max voltage & $4.15 \mathrm{~V}$ \\
\hline Min voltage & $2.5 \mathrm{~V}$ \\
\hline Nominal capacity & $20 \mathrm{Ah}$ \\
\hline Max discharge current (continuos) & $5 \mathrm{C}$ \\
\hline Max discharge current $($ peak $<10 \mathrm{~s})$ & $10 \mathrm{C}$ \\
\hline Operating temperature & $-30^{\circ} \mathrm{C} /+55^{\circ} \mathrm{C}$ \\
\hline
\end{tabular}




\begin{tabular}{|l|l|}
\hline Charging temperature & $0^{\circ} \mathrm{C} /+40^{\circ} \mathrm{C}$ \\
\hline Storage temperature & $-30^{\circ} \mathrm{C} /+55^{\circ} \mathrm{C}$ \\
\hline Specific energy & $174 \mathrm{Wh} / \mathrm{Kg}$ \\
\hline Volumetric energy density & $370 \mathrm{Wh} / \mathrm{L}$ \\
\hline Specific power (DoD $50 \%, 10 \mathrm{sec})$ & $2300 \mathrm{~W} / \mathrm{kg}$ \\
\hline $\begin{array}{l}\text { Volumetric power density (DoD } 50 \%, 10 \\
\text { sec) }\end{array}$ & $4600 \mathrm{~W} / \mathrm{L}$ \\
\hline Storage temperature & $-30^{\circ} \mathrm{C} /+55^{\circ} \mathrm{C}$ \\
\hline Specific energy & $174 \mathrm{Wh} / \mathrm{Kg}$ \\
\hline Volumetric energy density & $370 \mathrm{Wh} / \mathrm{L}$ \\
\hline
\end{tabular}

The equipment used for the life tests is summarized in Table 2. In particular, the table summarizes the main characteristics and the operating range of the bidirectional AC/DC converters (cyclers) used both in the cell formation phase and for the execution of life tests and tests of capacity and internal resistance, and of the climatic chambers in which the life tests and tests were conducted.

\begin{tabular}{|c|c|c|}
\hline Equipment & Name & Rating \\
\hline Cycler & ELTRA E-8094 (double field) & $\begin{array}{c}\mathrm{V}=0-36 \mathrm{VI}=280 \mathrm{~A} \\
\mathrm{Vmax}=36-52 \mathrm{VI}=400 \mathrm{~A}\end{array}$ \\
\hline Cycler & ELTRA E-8376 (double field) & $\begin{array}{l}V=0-35 \mathrm{VI}=400 \mathrm{~A} ; \\
V=36-350 \mathrm{VI}=600 \mathrm{~A}\end{array}$ \\
\hline Cycler & ELTRA E-8325 & $\begin{array}{l}\mathrm{V}=0-20 \mathrm{~V} ; \quad 80 \mathrm{~A} \quad \text { (charge)-150A } \\
\text { (discharge) }\end{array}$ \\
\hline Cycler & DIGATRON 80V 8 channels & $V=0-100 \mathrm{~V} \quad \mathrm{I}=50 \mathrm{~A}$ \\
\hline Cycler & $\begin{array}{l}\text { Maccor Series } 400048 \\
\text { channels }\end{array}$ & $\mathrm{V}=0-5 \mathrm{~V} \mathrm{I}=5 \mathrm{~A}$ \\
\hline Cycler & $\begin{array}{c}\text { Maccor Series } 40008 \\
\text { channels }\end{array}$ & $\mathrm{V}=0-80 \mathrm{~V} \mathrm{I}=50 \mathrm{~A}$ \\
\hline $\begin{array}{l}\text { Climatic } \\
\text { chamber }\end{array}$ & Angelantoni EOS 1000 & $-40^{\circ} \mathrm{C},+180^{\circ} \mathrm{C} ;$ U.R. $15-98 \%$ \\
\hline $\begin{array}{l}\text { Climatic } \\
\text { chamber }\end{array}$ & Angelantoni UY 2250 SP & $-40^{\circ} \mathrm{C},+180^{\circ} \mathrm{C} ;$ U.R. $15-98 \%$ \\
\hline $\begin{array}{l}\text { Climatic } \\
\text { chamber }\end{array}$ & Angelantoni DY 1200C EX & $-60^{\circ} \mathrm{C},+150^{\circ} \mathrm{C} ;$ U.R. $15-98 \%$ \\
\hline
\end{tabular}

For each cell subjected to the test, the following steps were carried out:

1) Initial inspection, to verify the absence of damage to the cell visible from the outside and the correspondence of the physical characteristics with those provided by the manufacturer. In our case, none of the tested cells were found to have any damage or discrepancies from the physical characteristics provided in the datasheet.

2) Electrical formation: consisting of a sequence of standard cycles (discharge at constant current (CC) at $\mathrm{C} / 2$, pause 1 hour, charge constant current-constant voltage (CC$\mathrm{CV}$ ) with current at $\mathrm{C} / 2$ and pause 1 hour) which ends when the discharge capacity relating to two consecutive discharges does not vary more than $3 \%$ of the value of the nominal capacity. The procedure ensures that the cells have reached an adequate stabilization of performance, before starting the actual test sequence.

3) Life cycles and periodic tests (see 3.1.1). 
4) Final inspection, to verify the presence of any damage and deformations that occurred during the tests.

\subsubsection{Life test procedures}

During the tests, various parameters are measured and recorded, among which are: date and time; battery voltage, current, capacity and energy; environment and battery temperature. The parameters are measured and recorded with a sufficiently high frequency to capture all relevant variations and make them available for further data processing. Depending on the technology of the cycler used, the detection takes place at a frequency established for each phase and/or when the variation of some parameters is higher than a certain limit.

Before each test, the cells are thermally stabilized through the use of a climatic chamber. To ensure that the initial conditions of the tests are always the same, at the beginning of each test a standard cycle is performed at the same temperature at which the life tests are performed $\left(\mathrm{T}=35^{\circ} \mathrm{C}\right)$.

After the standard cycle, life tests are performed, to highlight the link between the various cycling parameters studied and the decay curve of the performance of the cells being analyzed. The tests are periodically interrupted to carry out pre-established checks on the capacity and internal resistance.

The main parameters of the life tests are reported in Table 3. The cycles are balanced concerning the amount of charge delivered/accumulated. The average state of charge (SOC) of the battery is fixed at SOCav $=50 \%$ and the charging current at $0.5 \mathrm{C}$.

In tests from 1 to 4 , the stress for different discharge currents is compared (Crate $=1$, $2,3,5)$; the cells are cycled at $\Delta \mathrm{SOC}=60 \%(20 \% \leq \mathrm{SOC} \leq 80 \%)$.

In tests from 5 to 6 , different depth of discharge stresses are applied $(\triangle \mathrm{SOC}=80 \%$, $60 \%, 40 \%)$. The discharge current is equal to $1 \mathrm{C}$.

Tests 7-9 apply combinations of the above stresses. In particular, test 7 provides for $\Delta \mathrm{SOC}=40 \%$ and discharge current equal to Crate $=5 \mathrm{C}$, while test 8 provides for $\Delta \mathrm{SOC}=$ $80 \%(90 \% \leq \mathrm{SOC} \leq 10 \%)$ and discharge current equal to Crate $=3$.

To understand whether the model is able or not to predict the life span of the battery when it is subjected to variable stresses during its life, test 9 foresees aging cycles consisting in the repetition of a macrocycle formed by 8 cycles at one current with Crate $=3$ and $\triangle \mathrm{SOC}=80 \%$ and 10 cycles at a current with Crate $=2$ and $\triangle S O C=40 \%$, where the duration of each macrocycle is two days.

\begin{tabular}{|c|c|c|c|}
\hline Test number & $\begin{array}{c}\text { Discharge current } \\
\text { (C-rate) }\end{array}$ & $\begin{array}{c}\Delta \text { SOC=SOCin- } \\
\text { SOCfin }\end{array}$ & $\begin{array}{c}\text { Cycle between } \\
\text { control tests }\end{array}$ \\
\hline 1 & $1 \mathrm{C}$ & $80-20$ & 200 \\
\hline 2 & $2 \mathrm{C}$ & $80-20$ & 200 \\
\hline 3 & $3 \mathrm{C}$ & $80-20$ & 1001 \\
\hline 4 & $5 \mathrm{C}$ & $80-20$ & 100 \\
\hline 5 & $1 \mathrm{C}$ & $90-10$ & 160 \\
\hline 6 & $1 \mathrm{C}$ & $70-30$ & 320 \\
\hline 7 & $5 \mathrm{C}$ & $70-30$ & 320 \\
\hline 8 & $3 \mathrm{C}$ & $90-10$ & 160 \\
\hline 9 & $8 \times 3 C @ 80 \% \mathrm{DOD}+$ & $90-10,70-30$ & 90 \\
\hline & $10 \times 2 C @ 60 \% D O D$ & & \\
\hline
\end{tabular}

\footnotetext{
1 First control test after 200 cycles.
} 
Each test is associated with a given cell, identified by a progressive number. The last column shows the intervals between the periodic control tests of the decay of performance in terms of the number of life cycles performed.

Of all the batteries analyzed, only the n. 2 presented a change in shape in the final inspection, due to a swelling of the envelope. The cell thickness went from the initial value of $7.2 \mathrm{~mm}$ to the final value of $10.4 \mathrm{~mm}$, with a percentage variation of about $40 \%$. After the suspension of the life tests, there was no further increase in swelling, nor a regression of the same.

\subsubsection{Control test procedure}

The control test procedure is carried out in a climatic chamber at a temperature of $20{ }^{\circ} \mathrm{C}$. Although the control test involves the measurement of capacity and internal resistance, the latter parameter is not used in the methodology we present, and will therefore not be presented in this discussion.

The capacitance measurement test consists of measuring the capacitance value of a cell, after a thermal stabilization pause, by using a standard cycle. The cell is discharged CC at $\mathrm{I}=0.5 \mathrm{C}$, rested for 30 minutes and then subjected to a CC-CV charge at $\mathrm{I}=0.5 \mathrm{C}$ up to $\mathrm{SOC}=100 \%$. The procedure is repeated for a current of $1 \mathrm{C}$. Each of these processes is interspersed with appropriately calibrated pauses. The test is repeated twice with each measurement session.

\subsection{Modeling of cell behavior}

To compare the experimental results for life-tests in which the definition of the cycle is different in duration and depth of discharge, it is necessary to find a suitable parameter. It was therefore considered appropriate to analyze the capacity trend as a function of the cumulative charge, defined by the equation:

$$
A h_{t}=\int_{0}^{t}\left|I\left(t^{\prime}\right)\right| d t^{\prime}
$$

Where $\mathrm{I}\left(\mathrm{t}^{\prime}\right)$ is the instantaneous current flowing through the cell. With this variable, the capacity trends for cells whose duty cycles have different depths of discharge can be compared.

Figure 2 graphically shows the relationship between the amount of cumulative charge supplied until reaching the end of life condition $(C / C 0=0.8)$ and the stress factors analyzed, for cells B1 - B8. Experimental points are represented as black dots on the surface.

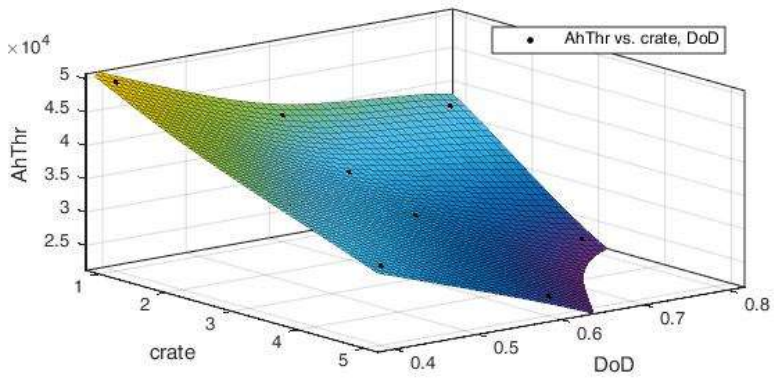

(a)

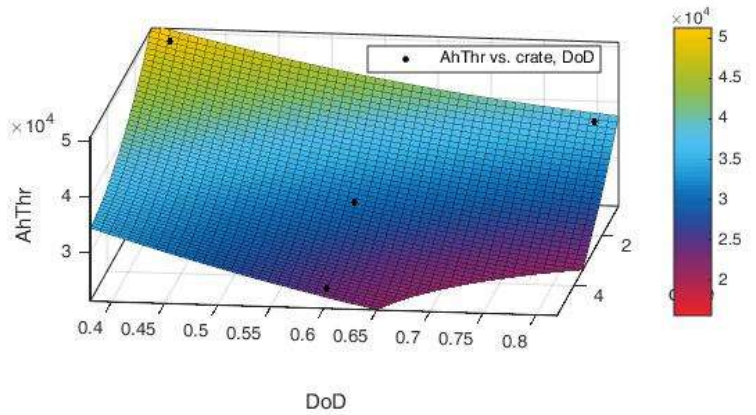

(b)

Figure 2: Graphical representation of the cumulative charge trend as a function of the C-rate and the depth of discharge of the life cycles. 
The curve was interpolated with a second-degree polynomial function with respect to the two independent variables, using the MatLab® Curve Fitting Toolbox. The value of $\mathrm{R} 2$ is very close to 1 , but the value of the sum of squares due to error (SSE) is extremely high, which means that the model has a big random error component and that the fit will is not useful for prediction.

\subsubsection{Proposed model}

In its simplest form, an aging model consists of an empirical correlation between the observable quantities (capacitance and/or internal resistance) as a function of time or cycles or cumulative charge, and various aging factors, such as temperature, current, state of charge, etc.

In the present study, we want to find a model that describes the change of the relative value of the battery capacity defined as:

$$
C_{r}(x)=\frac{C(x)}{C(0)}
$$

Where $C(0)$ is the value of the initial (or nominal) capacity of the battery, and $C(x)$ is the value of the capacity measured at a given time $t$ which is a function of the cumulative charge $x=A h_{t}$

The proposed model has its base on the work by Risse et al. [24]. In this work, the battery capacity degradation process is represented by a Markov chain consisting of three phases and four states, capable of capturing the phenomena that occur during the aging of a battery, including initial formation. The proposed model can be generalized to different types of batteries and is very flexible. In the present work, we are not interested in the formation phase, so we can reduce the model to three phases: the sleeping phase $f_{s}$, which represents the portion of the bound charge that can be converted into the active phase during formation and cycling; the living phase $f_{l}$, represents the capacity immediately available; the dead phase $f_{d}$ that represent the portion of material no longer available for cycling. The system state is a combination of the three phases. Once the active materials are transferred from the active state to the dead state, they can no longer be used in the charging and discharging processes. The dead state is thus an absorbing state. The state of the system is represented as a combination of the single states.

At each step, each phase A evolves into phase B with probability $P(A \rightarrow B \mid A) P(A)$, where $P(A) \stackrel{\text { def }}{=} f_{A}(n)$ is the probability of finding the system in state $\mathrm{A}$ and coincides with the fraction of the system that is in that state. $P(A \rightarrow B \mid A) \triangleq k_{A \rightarrow B}$ is the conditional probability that the system passes from state A to state $B$. The model describes the evolution of the fractions of the phases $f_{i}$ after a charge and discharge cycle by means of a suitable transition matrix between the states:

$$
T=\left(\begin{array}{ccc}
1-k_{l \rightarrow d} & k_{s \rightarrow l} & 0 \\
0 & 1-k_{s \rightarrow l} & 0 \\
k_{l \rightarrow d} & 0 & 1
\end{array}\right)
$$

Where $k_{l \rightarrow d}, k_{s \rightarrow l}$ are the transition probabilities between the living and dead phase and between the sleeping and living phase of the model, respectively. The Markov chain described by the transition matrix is stationary. Furthermore, the sum of all elements of the state vector remains constant, ensuring the conservation of mass in the process.

The state of the system at cycle $n$ is represented by a column vector whose elements represent the fraction in the three different phases:

$$
\overrightarrow{f_{n}}=\left\{f_{l}(n), f_{s}(n), f_{d}(n)\right\}^{T}
$$

Starting from an initial state $\overrightarrow{f_{0}}=\left\{f_{l, 0}, f_{s, 0}, f_{d, 0}\right\}^{T}$, the transition from the $\mathrm{n}$-th cycle to the $\mathrm{n}$ + 1-th cycle is obtained by multiplying the vector by the transition matrix:

$$
\overrightarrow{f_{n+1}}=T \overrightarrow{f_{n}}=T^{n+1} \overrightarrow{f_{0}}
$$


From the previous definitions, it can be deduced that the measured capacity is proportional to the fraction of the system state that is in the active phase. Applying formula (4), the active phase after $n$ cycles is given by:

$$
\mathrm{P}_{\text {live }}(\mathrm{n})=f_{l, 0}\left(1-k_{l \rightarrow d}\right)^{\mathrm{n}}+f_{s, 0} k_{s \rightarrow l}\left(\frac{\left(1-k_{l \rightarrow d}\right)^{\mathrm{n}}-\left(1-k_{S \rightarrow l}\right)^{\mathrm{n}}}{k_{s \rightarrow l}-k_{l \rightarrow d}}\right)
$$

When we try to fit the experimental data with the Markov model, we find that the model is able to reproduce the degradation trend of the batteries only when the intensity of the applied stresses is not too high, while deviations are detected between the model fitting and the experimental data for higher intensities of stress. In particular, the model fails to reproduce the capacity degradation curve when it shows a variation in the slope that usually occurs as the strength of the applied stresses increases. As an example, we report some degradation curves (in blue) and the fitting of the Markov model (in red) in Error! Reference source not found. for battery 2 (a), subjected to a charge current of $2 \mathrm{C}$ and $\triangle S O C$ of $60 \%$, and battery 8 (b), subjected to a current of $3 C$ and $\triangle S O C$ of $80 \%$.

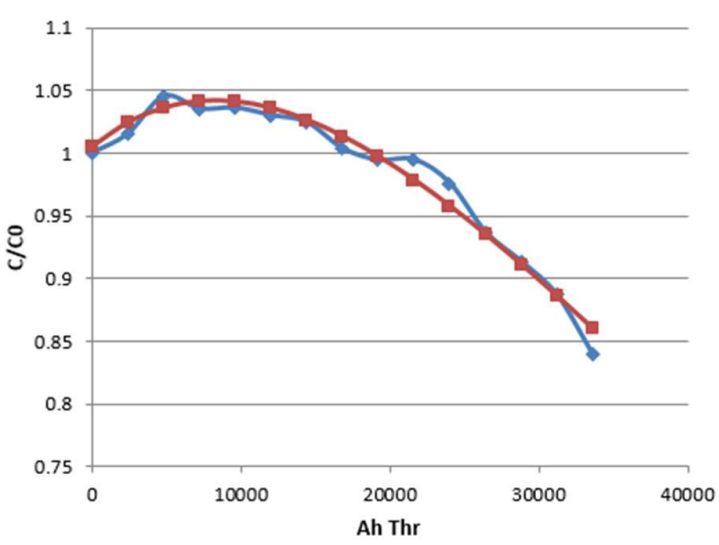

(a)

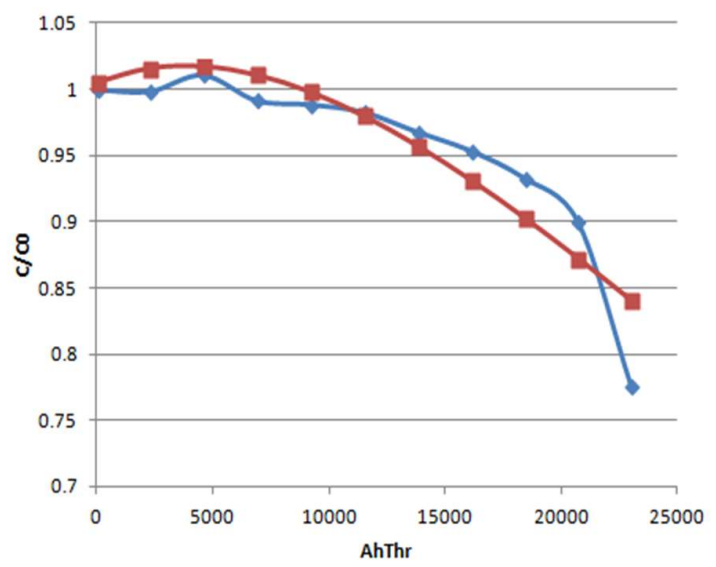

(b)

Figure 3: Comparison between the trend of the experimental and Markov degradation curve for battery 2 (a) and for battery 8 (b). Blue line, diamond symbol: experimental data; red line square symbol: Markov fitting.

As it can be seen from Figure 3 (a), for the battery with lower stress intensity, the Markov model is able to catch the main features of the experimental curve. However, the deviation from experimental data is relevant in the case of stronger stress, especially toward the end of life (Figure $3(b))$.

In order to interpolate the experimental data it is necessary to modify the model. In particular, to describe the knee present in the degradation curve, a transition probability has been introduced which depends on the number of suitably defined cycles $\mathrm{n}$. The transition matrix at cycle $\mathrm{n}$ is represented in formula (7):

$$
Q^{n}=\left(\begin{array}{ccc}
1-a(n / d)^{e}-b & c & 0 \\
0 & 1-c & 0 \\
a(n / d)^{e}+b & 0 & 1
\end{array}\right)
$$


The parameters $a, b, c, d$ and e are determined from the experimental data. Since the transition probability depends on the system hystory, the resulting model is non-markovian. The state of the system is represented as before by the weighted combination of the three possible phases:

$$
\overrightarrow{f_{n}}=\left\{f_{l}(n), f_{s}(n), f_{d}(n)\right\}^{T}=Q^{n} \overrightarrow{f_{0}}
$$

The trend of the capacity is represented by the component of the state that corresponds to the living phase $f_{l}(n)$. The transition matrix (7) reduces to that of the original Markov model when the term $a(n / d)^{e} \rightarrow 0$.

Unlike the Markov model (3), for the introduced model it is not possible to find a closed analytic expression for $f_{l}(n)$, so it is necessary to resort to a numerical solution. MatLab® was used to solve the problem of determining the parameters of the transition matrix and of the initial phases value.

As model (7) contains the number of cycle in its definition, it represents a discrete process. In order to compare the results for cells subjected to life tests with different $\triangle S O C \mathrm{~s}$, it is necessary to appropriately quantize the cumulative charge, to obtain a suitably defined equivalent cycle. To this end, we consider the $\triangle \mathrm{SOC}$ of the life tests and take the greatest common factor (GCF). We then define the number of equivalent cycle ce of each life test as the number of time the GCF enters the $\triangle$ SOC. In our case, we considered $3 \triangle S O C: 40 \%$, $60 \%$ and $80 \%$. The GCF is therefore worth $20 \%$. Hence, the number of ce equals 2, 3 and 4 for each cycle at $\Delta$ SOC $40 \%, 60 \%$ and $80 \%$, respectively. Obviously, this is not the only quantization possible, but a different choice does not affect the validity of the model, although it would lead to different results for the parameters, as long as the transformation is linear.

We report the results obtained with this model for batteries 1-4 in Table 2.

Table 2: Parameters of the model (7) for batteries with different C-rate as a function of the number of equivalent cycles

\begin{tabular}{|l|l|l|l|l|}
\hline $\begin{array}{l}\text { Model } \\
\text { parameters }\end{array}$ & B1 & B2 & B3 & B4 \\
\hline fl & 1.01 & 1.008 & 1.009 & 1.04 \\
\hline fs & 1.114 & 1.016 & 1.036 & 1.185 \\
\hline a & 0.0001846 & 0.0003431 & 0.000104 & 0.0001705 \\
\hline b & $8.733 \mathrm{e}-05$ & $9.388 \mathrm{e}-05$ & 0.000107 & $9.571 \mathrm{e}-05$ \\
\hline c & $9.667 \mathrm{e}-05$ & 0.0001235 & 0.0001035 & $8.01 \mathrm{e}-05$ \\
\hline $\mathrm{d}$ & $1.003 \mathrm{e}+04$ & 9200 & 7138 & 5609 \\
\hline $\mathrm{e}$ & 14.86 & 10.98 & 15.88 & 26.54 \\
\hline R & 0.994 & 0.9873 & 0.9842 & 0.9496 \\
\hline
\end{tabular}

However, the large number of parameters makes the interpretation of the results complex, and the model is more prone to numerical errors. Since the values of $f_{l}(0)$ and $f_{s}(0)$ are 
almost constant for all batteries, as also happens in the original model (3), we replace these parameters with the average values obtained, that is:

$f_{l}(0) \equiv f_{l 0}=1.005$;

$f_{s}(0) \equiv f_{s 0}=1.1$.

The parameters $f_{l}(0)$ and $f_{s}(0)$ have intrinsic physical meaning for the cells since the parameters represent the cells' initial state, which should depend mainly on cell composition. To verify the influence of the parameters $f_{l}(0)$ and $f_{s}(0)$ on the model output, we made a series of simulations where the values of the two parameters are randomly chosen from two independent Gaussian distributions, with average value centered in $f_{l}(0)$ and $f_{s}(0)$, respectively, and relative variance of 0.03 . All the other parameters remained fixed at the value determined by the MatLab tool for $f_{l}(0)=f_{l 0}$ and $f_{s}(0)=f_{s 0}$.

The results are shown in Figure 4 for the B2 battery. The simulation was made for a sample of 100 initial values of $f_{l}(0)$ and $f_{s}(0)$ and, for comparison, the experimental curve is also shown (in green with round symbols). It can be seen that the curves tend to converge as the number of equivalent cycles increases, indicating a limited influence of the values of the two parameters in the final part of life (variation of less than $2 \%$ in the cases examined). We can therefore consider that the error introduced by setting the value of $f_{l}(0)$ and $f_{s}(0)$ is irrelevant for determining the end of life.

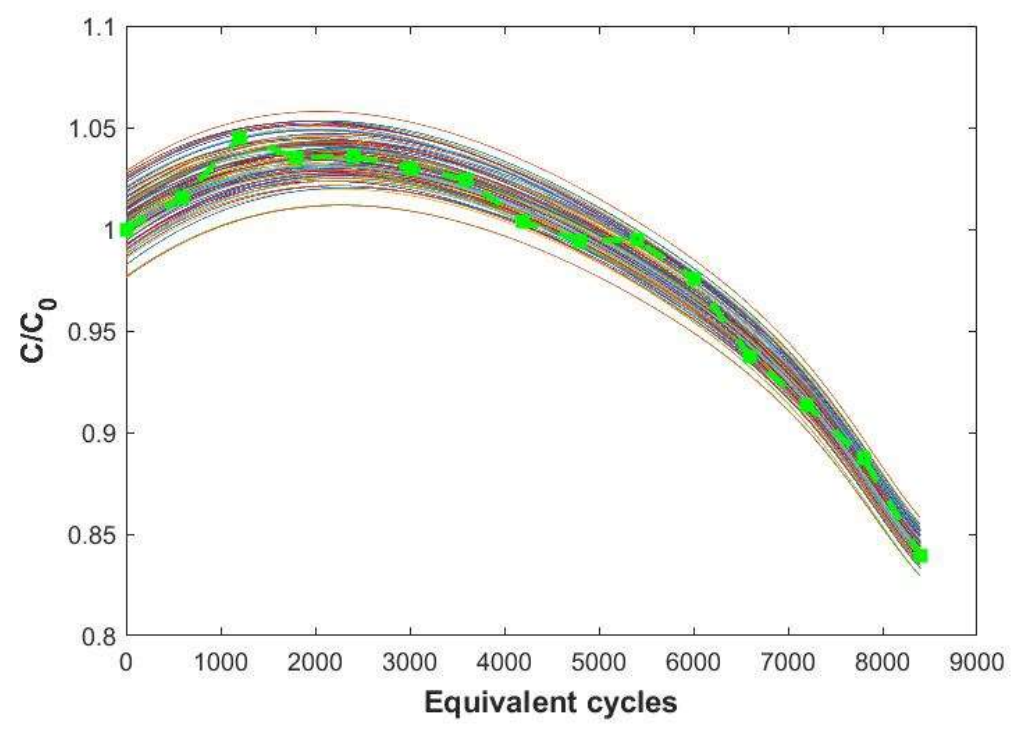

Figure 1: Effects of the variation of $\mathrm{fl} 0$ and $\mathrm{fs} 0$ on the capacity trend as a function of the number of equivalent cycles. In green with round symbols, the experimental curve for B2.

In Table 5 we report the parameter values for batteries aged at different discharge currents for the model (7) with fixed $f_{l 0}$ and $f_{s 0}$. The discrepancy for the battery 2 in the trend for the value of the exponent e (also reported in Table 4) could depend on the fact that the battery has undergone a swelling before the end of its life, to signal some type of internal malfunction that has been highlighted in the post-mortem analyzes [25]. 
Table 5: Model parameter with fixed values for $f_{l 0}$ and $f_{s 0}$ for batteries with different C-rate

\begin{tabular}{|l|l|l|l|l|}
\hline Model parameters & B1 & B2 & B3 & B4 \\
\hline a & 0.0001713 & 0.0003379 & 0.0001123 & 0.0001705 \\
\hline b & $8.847 \mathrm{e}-05$ & $9.762 \mathrm{e}-05$ & 0.000112 & 0.0001189 \\
\hline c & 0.0001018 & 0.0001183 & 0.0001298 & 0.0001331 \\
\hline d & 9970 & 9175 & 7172 & 5669 \\
\hline e & 16.43 & 10.19 & 16.74 & 36.66 \\
\hline R $^{2}$ & 0.994 & 0.9873 & 0.9842 & 0.9496 \\
\hline
\end{tabular}

The same model was applied to the data obtained for batteries aged with different $\triangle$ SOCs. The results are reported in Table 6.

Table 6: Model parameter with fixed values for $f_{l 0}$ and $f_{s 0}$ for batteries with different $\Delta \mathrm{SOC}$

\begin{tabular}{|l|l|l|l|}
\hline Model parameters & B6 & B1 & B5 \\
\hline a & 0.0001013 & 0.0001713 & 0.0001379 \\
\hline b & $4.993 \mathrm{e}-05$ & $8.847 \mathrm{e}-05$ & $9.817 \mathrm{e}-05$ \\
\hline c & $5.802 \mathrm{e}-05$ & 0.0001018 & 0.000112 \\
\hline d & $1.139 \mathrm{e}+04$ & 9970 & 9844 \\
\hline e & 8.65 & 16.43 & 44.15 \\
\hline R $^{2}$ & 0.9777 & 0.994 & 0.9844 \\
\hline
\end{tabular}

As for the multiple stress tests carried out on batteries 7 and 8 , the results obtained are shown in Table 7.

Table 7: Model parameter with fixed values for $f_{l 0}$ and $f_{s 0}$ for batteries with multiple stress

\begin{tabular}{|l|l|l|}
\hline Model parameters & B7 & B8 \\
\hline a & $9.021 \mathrm{e}-05$ & 0.0002348 \\
\hline b & $4.396 \mathrm{e}-05$ & $9.183 \mathrm{e}-05$ \\
\hline c & $4.583 \mathrm{e}-05$ & $8.713 \mathrm{e}-05$ \\
\hline d & 7202 & 6086 \\
\hline e & 8.203 & 17.31 \\
\hline R $^{2}$ & 0.9818 & 0.9964 \\
\hline
\end{tabular}

By way of example, Figure 5 (a) shows the comparison between the experimental trend of degradation for battery 2 and the results obtained from the model (10) as a function of the number of equivalent cycles. The model captures the performance of the battery better than the Markov model in the final phase of the curve. 


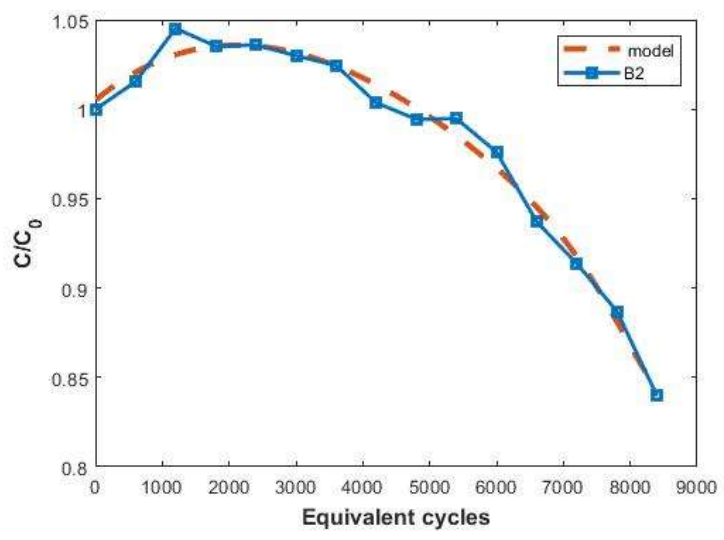

(a)

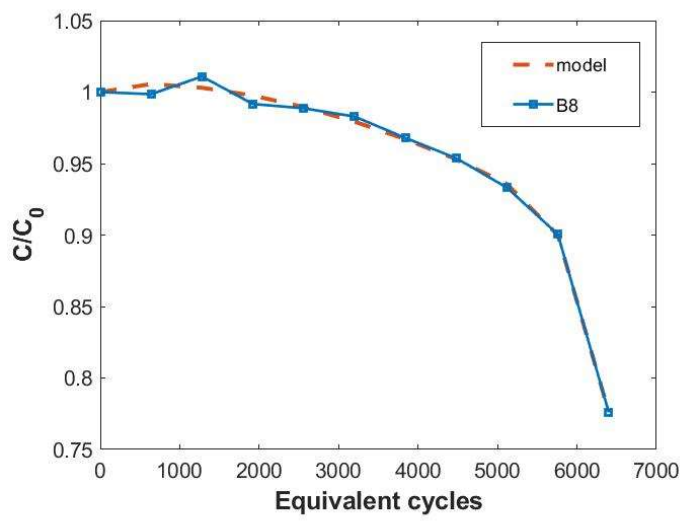

(b)

Figure 5: Comparison between the experimental data and the model (7) for batteries 2 and 8 as a function of the number of equivalent cycles

Figure 5 (b) shows the degradation curve obtained for battery 8 with the model (7), together with the experimental data. Comparing it with the curve in Figure 3 it is clear that the modified model is able to reproduce the less regular behavior of cells subjected to high-stress intensities. In particular, the knee present in the experimental curve is captured by the proposed model.

\subsubsection{Predictive efficacy of the model}

In this section we want to verify the model's ability to predict the battery life when it is subjected to a work cycle composed of different stress intensities, using the results of the parameters obtained for laboratory tests conducted with stresses of predefined intensity. For this purpose, battery 9 was subjected to a life test whose aging cycle consists of the repetition of a macrocycle composed as follows:

a) 8 cycles at a current rate of $\mathrm{Crate}=3$ and $\triangle \mathrm{SOC}=80 \%$;

b) 10 cycles at a current rate of Crate $=2$ and $\triangle \mathrm{SOC}=60 \%$.

The number of equivalent cycles that make up the macrocyle is composed of $8 * 4=32$ equivalent cycles for the Crate phase $=3$ and $\triangle \mathrm{SOC}=80 \%$, and $10 * 3=30$ equivalent cycles for the Crate $=2$ phase and $\triangle S O C=60 \%$, for a total of 62 equivalent cycles per macrocycle.

The results for the degradation curve of battery 9 are reported in Figure 6. For comparison, the experimental data of the batteries 8 and 2, which have been subjected to aging cycles of type a) and b) respectively, are also reported. If the effect of the different stress factors were added linearly, the degradation curve of battery 9 should be between those of batteries 8 and 2. However, the experimental data show that the curve is rather flattened on that of battery 8 . 


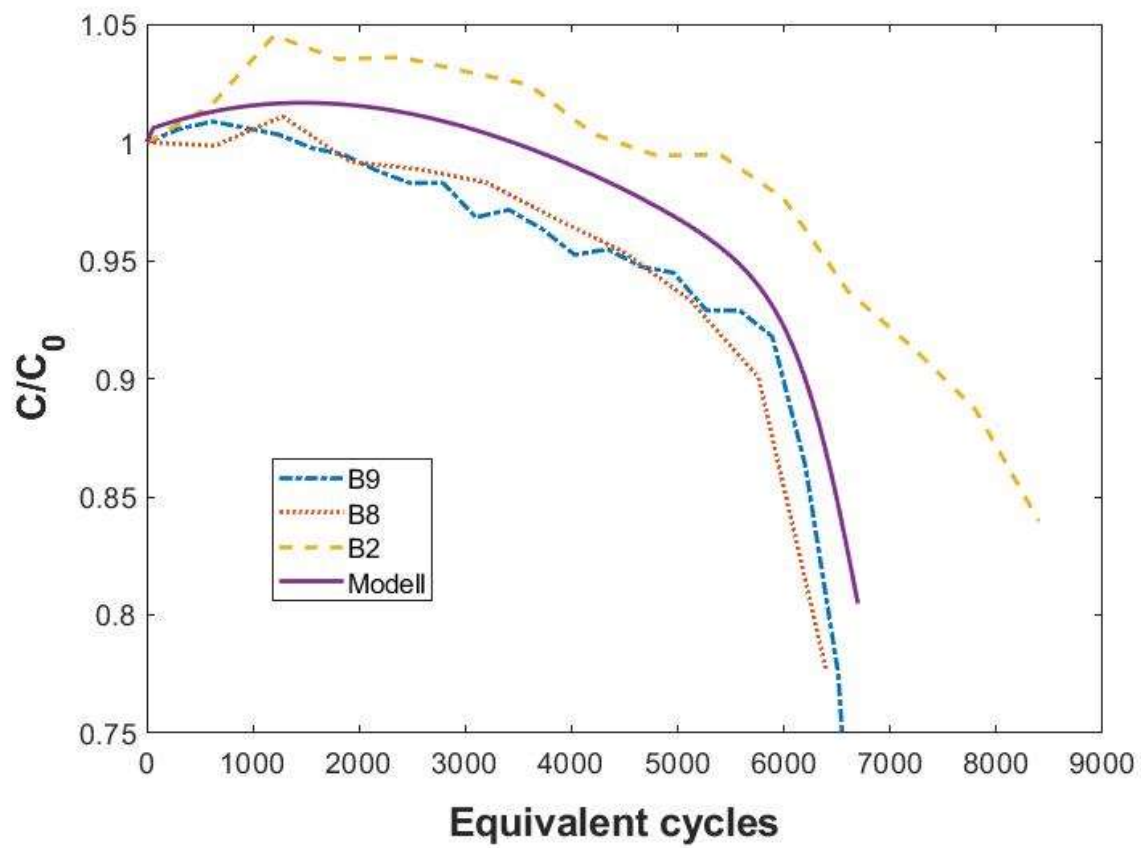

Figure 2: Degradation curve of the capacity for cell b9 with respect to the number of equivalent cycles and comparison between the predictions of the model (7). For comparison, the curves of batteries 8 and 2 are shown.

In Figure 2 the results of the life simulation obtained by applying the model were also reported (7). Figure 7 shows the flowchart of the program: as input, we have the parameters from the experimental data for $\mathrm{B} 2$ and $\mathrm{B} 8$, as well as the fixed values of $f_{l 0}$ and $f_{s 0}$. These represent the initial values of the vector $\mathrm{q}$, which coincides with the initial state of the system $\overrightarrow{f_{0}}$ whose third component, not shown, represents the dead phase. The parameters of the batteries B8 and B2 were appropriately inserted in the transition matrix (7) which was then used in the algorithm, represented by the loops shown in the flowchart. The output, is the capacity of the battery after $n_{e q}$ equivalent cycles. In this way, it is possible to obtain the simulation of the decay of the capacitance of B9. 


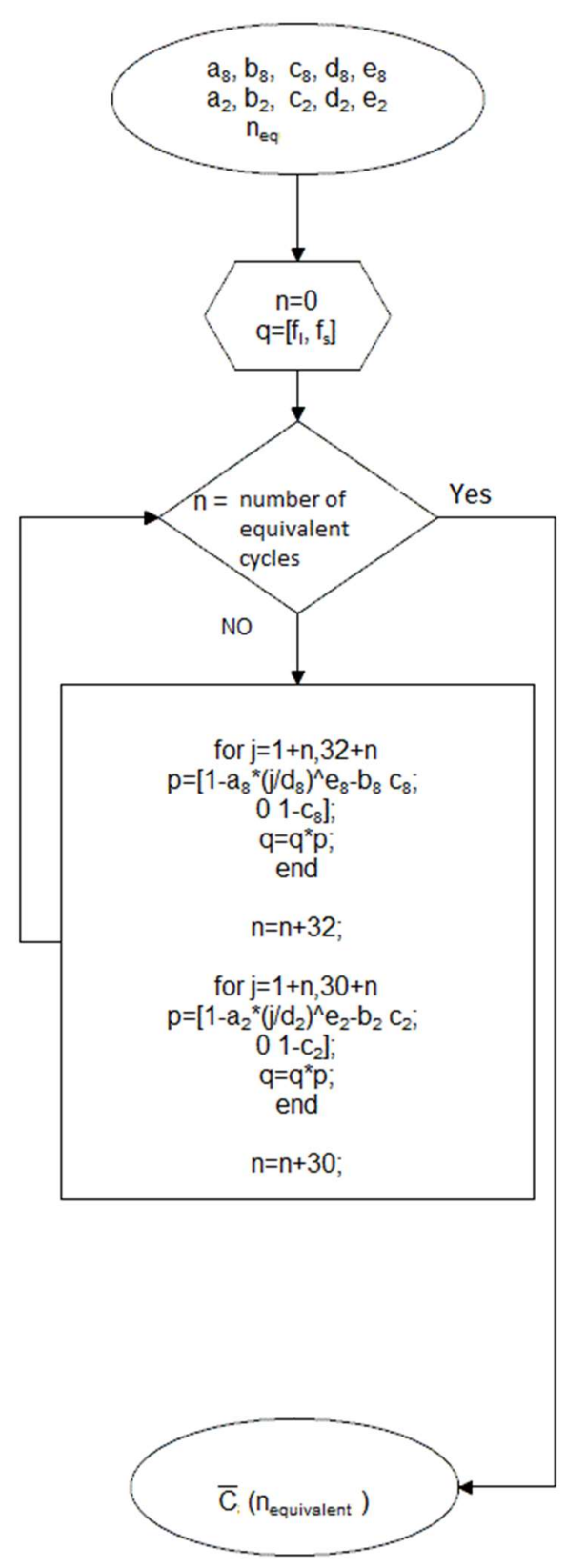

Figure 3: Flowchart of the life span simulation program based on the model (7).

However, capacitance measurements are subject to many errors which introduce a source of uncontrollable variability. These variations can arise from several sources:

1. Inherent System Uncertainties: Due to uncertainties in manufacturing assembly and material properties, batteries may have different initial capacities. Each battery can also be individually affected by impurities or defects, which can lead to different aging rates [26].

2. Measurement uncertainties: Uncertainties are likely to arise from the background noise of measurement devices.

3. Uncertainties in the operating environment: the rate of capacity fade may be affected by conditions of use such as a shorter or longer shelf life before testing. 
4. Modeling uncertainties: the model is an approximation of battery degradation, which will lead to some modeling errors.

To partially obviate the random errors highlighted in points 1) -3) above, let's analyze the model response when adding white noise to the model parameters. Analyzing the response for batteries 1-8, it is highlighted that the most dramatic effects for the evaluation of the battery life are due to the uncertainty on the parameters $a, d$, and e, which particularly affect the final part of the curve, making, therefore, it is more difficult to estimate the life of the batteries.

By applying this process to all the parameters of batteries 2 and 8 and averaging the results obtained from the simulation, we obtain a curve that is closer to the experimental one in the end-of-life area, compared to that obtained using only the parameters extracted from the experimental results of B2 and B8. This result reinforces the fact that a more substantial statistic of experimental data could help to better identify the range of validity of the predictions. In Figure 8 the experimental curve (yellow), the model output (blue) and the averaged output over the uncertain (red) are reported.

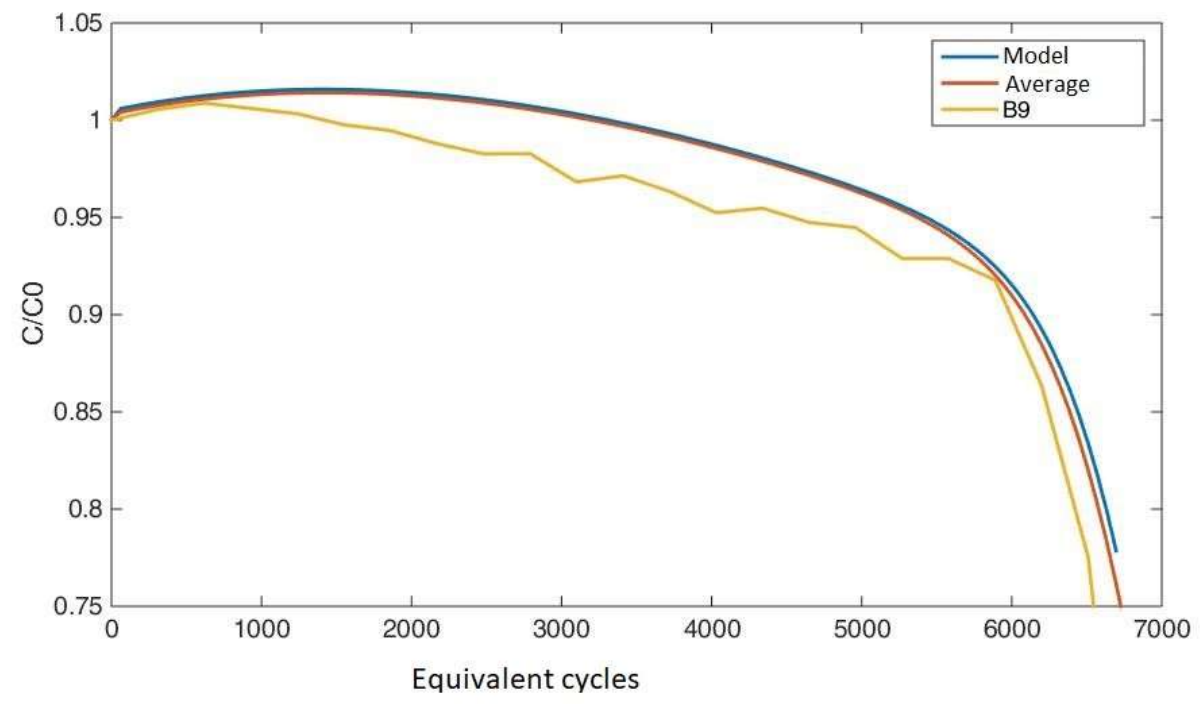

Figura 4: Comparison between the experimental curve, the estimate of the modified Markov model and the simulation average.

The percentage errors on the estimate of the cell life span obtained with this model, compared to the number of equivalent cycles when the relative capacity has reached 0.78 , are reported in Table 8.

Table 3: Error on the estimation of the number of end-of-life cycles of the model (7) and of the average of the simulations with Gaussian distribution of the model parameters.

\begin{tabular}{|l|l|l|l|}
\hline B9 & Experimental & Model (7) & $\begin{array}{l}\text { Averaged value } \\
\text { over uncertains }\end{array}$ \\
\hline $\begin{array}{l}\text { No. Equivalent } \\
\text { cycles }\end{array}$ & 6510 & 6696 & 6634 \\
\hline Error \% & - & 2.8 & 1.9 \\
\hline
\end{tabular}

\section{Conclusions}


The present study aims at determining the effects that some stress factors, such as depth of discharge and discharge current, have on the cycle life of lithium-ion batteries. To this end, life tests were conducted on battery cells. In particular, the cells under consideration use a technology based on lithium nickel cobalt manganese oxide (NCM) at the positive electrode and graphite at the negative electrode. Since the anode and cathode compositions are fundamental in the determination of the degradation process of lithium-ion cells, the results obtained during the study are strictly valid only for the analyzed technology. The results obtained are in any case in line with what is known in scientific literature, i.e. a faster degradation of batteries subjected to high discharge currents and greater depths of discharge.

We set up an experimental matrix to evaluate the impact of different stresses on the capacity fade. The data were used to study a methodology that would allow reasonable life estimation even with a limited number of data available. We focused on a model that refers to the Markovian process structure, although it is not Markovian since the probability transition is dependent on the system history. This model describes the evolution of the system starting from an initial state consisting of different phases that evolves into successive states through a transition matrix. The three possible states are: dormant, in which the potential charge is found but not directly available for cycling; active, in which the charge available for cycling is found; dead, in which the charge is no longer available. The latter is an absorber state of the system, that is, the fraction of the system that reaches this state cannot pass into any other state. This model showed a good degree of fitting for the degradation curves of the analyzed cells, also managing to capture the knee present in some experimental results. As regards the ability to predict the trend of the capacity degradation curve when analyzing a cell subjected to mixed stress starting from a grid of results obtained at constant stresses, the proposed model is able to estimate the battery life with an error of about 3\% for NMC-graphite cells. Considering that the model parameters were extracted from data sets obtained from single tests for each stress, the result can be regarded as satisfactory, although it is expected to improve by increasing the data statistic. 
Author Contributions: Conceptualization, N.A.; methodology, N.A.; software, N.A.; validation, N.A., F.V. and V.S.; formal analysis, N.A.; investigation, N.A. and V.S..; resources, N.A and F.V.; data curation, N.A; writing-original draft preparation, N.A.; writing-review and editing, N.A and F.V..; visualization, N.A.; supervision, N.A; project administration, N.A.; funding acquisition, N.A. All authors have read and agreed to the published version of the manuscript.

Funding: This research was funded by Italian Ministry of Economic Development, under the project PTR 2019-21 of the Electrical System Research, Project 1.7 "Technologies for the efficient penetration of the electric vector in end uses", WP2 "Mobility".

Conflicts of Interest: The authors declare no conflict of interest

\section{References}

1. Venet, P.; Redondo-Iglesias, E. Batteries and Supercapacitors Aging. Batteries 2020, 6, 18. https://doi.org/10.3390/batteries601001

2. Tang, X.; et al. Aging trajectory prediction for lithium-ion batteries via model migration and Bayesian Monte Carlo method. Applied Energy 2019, 254, 113591.

3. Li, Y., et al., Data-driven health estimation and lifetime prediction of lithium-ion batteries: A review. Renewable and Sustainable Energy Reviews 2019, 113, 109254.

4. Zheng, Y.; Ouyang, M.; Lu, L.; Li, J. Understanding aging mechanisms in lithium-ion battery packs: From cell capacity loss to pack capacity evolution. Journal of Power Sources 2015, 278, 287. https://doi.org/10.1016/j.jpowsour.2014.12.105.

5. Jafari, M.; Khan, K.; Gauchia, L. Deterministic models of Li-ion battery aging: It is a matter of scale. Journal of Energy Storage 2018. 20, 2018, 67. https://doi.org/10.1016/j.est.2018.09.002.

6. Barré, A.; et al. A review on lithium-ion battery ageing mechanisms and estimations for automotive applications. Journal of Power Sources 2013, 241, 680. https://doi.org/10.1016/j.jpow$\underline{\text { sour.2013.05.040 }}$

7. Mukhopadhyay, A.; Sheldon, B. W. Deformation and stress in electrode materials for Li-ion batteries. Progress in Materials Science 2014, 63, 58.

8. Vetter, J.; et al. Ageing mechanisms in lithium-ion batteries, Journal of Power Sources 2005, 147 269.

9. Werner, D.; Paarmann, S.; Wetzel, T. Calendar Aging of Li-Ion Cells-Experimental Investigation and Empirical Correlation. Batteries 2021, 7, 28. https://doi.org/10.3390/batteries7020028

10. Redondo-Iglesias, E.; Venet, P.; Pelissier, S. Modelling Lithium-Ion Battery Ageing in Electric Vehicle Applications-Calendar and Cycling Ageing Combination Effects. Batteries 2020, 6, 14. https://doi.org/10.3390/batteries6010014

11. Leng, F.; Tan, C.; Pecht, M. Effect of Temperature on the Aging rate of Li Ion Battery Operating above Room Temperature. Sci. Rep. 2015, 5, 12967 . https://doi.org/10.1038/srep12967

12. Alipour, M.; Ziebert, C.; Conte, F.V.; Kizilel, R. A Review on Temperature-Dependent Electrochemical Properties, Aging, and Performance of Lithium-Ion Cells. Batteries 2020, 6, 35. https://doi.org/10.3390/batteries6030035

13. International Organization for Standardization. Available on line: https://www.iso.org/standard/71407.html (accessed on 26/05/2021)

14. Ning, G.; Popov, B. N. Cycle life modeling of lithium-ion batteries, Journal of The Electrochemical Society 2005, 151, 1584.

15. Doyle, M.; Fuller, T.F.; Newman, J. Modeling of galvanostatic charge and discharge of the lithium/polymer/insertion cell. Journal of the Electrochemical Society 1993, 140 (6), 1526-1533.

16. Doyle, M.; Newman, J. Analysis of capacity-rate data for lithium batteries using simplified models of the discharge process. Journal of Applied Electrochemistry 1997, 27, 846. https://doi.org/10.1023/A:1018481030499

17. Doyle, M., Fuller, T.F.; Newman, J. Relaxation phenomena in lithium-ion-insertion cells. Journal of the Electrochemical Society 1994, 141, 982.

18. Madani, S.S.; Schaltz, E.; Kær, S.K. A Review of Different Electric Equivalent Circuit Models and Parameter Identification Methods of Lithium-Ion Batteries. ECS Trans. 2018, 87, 23.

19. Delacourt, C.; Safari M. Mathematical Modeling of Aging of Li-Ion Batteries. In: Physical Multiscale Modeling and Numerical Simulation of Electrochemical Devices for Energy Conversion and Storage. Green Energy and Technology. Franco, A., Doublet, M., Bessler, W., Eds.; Springer: London, UK, 2016, pp. 151-190. 
20. Andrenacci, N.; Sglavo, V.; Vellucci, F. Stato dell'arte dei modelli di invecchiamento per le celle litio-ione. Applicazione al caso di studio delle celle NMC invecchiate in ENEA. Report RDS/ PAR2016/163 Avilable on line: https://www.enea.it/it/Ricerca_sviluppo/lenergia/ricerca-disistema-elettrico/accordo-di-programma-MiSE-ENEA-2015-2017/trasmissione-e-distribuzionedellenergia-elettrica/sistemi-di-accumulo-di-energia/report-2016 (accessed on 25/05/2021, in It.)

21. Chang, C-Y.; Tulpule, P.; Rizzoni, G.; Zhang, W.; Du, X. A probabilistic approach for prognosis of battery pack aging, Journal of Power Sources 2017, 347, 57. https://doi.org/10.1016/j.jpowsour.2017.01.130.

22. Jiang, L.; et al. Analysis of Data-Driven Prediction Algorithms for Lithium-Ion Batteries Remaining Useful Life. Advanced Materials Research 2013, 717, 390.

23. Chiodo, E.; Lauria, D.; Mottola F.; Andrenacci, N. On line Bayes Estimation of Capacity Fading for Battery Lifetime Assessment, Proceeding of 2019 International Conference on Clean Electrical Power (ICCEP), Otranto, Italy, 2019, pp. 599-604.

24. Risse, S.; Angioletti-Uberti, S.; Dzubiella, J.; Ballauff, M. Capacity fading in lithium/sulfur batteries: a linear four-state model. Journal Power Sources 2014, 267, 54.

25. Bacaloni, A.; et al. Characterization of Li-ion batteries for safety and health protection, IJOEHY 2019, 10, 1

26. Harris, S.J.; Harris, D.J.; Li, C. Failure statistics for commercial lithium ion batteries: A study of 24 pouch cells. Journal Power Sources 2017, 342, 589. 